\title{
Neuronal adaptation in the course of the prolonged task improves visual stimuli processing
}

\author{
Vladimir Maksimenko ${ }^{1,2, *}$, Alexander Kuc ${ }^{2}$, Nikita S. Frolov ${ }^{2}$, Alexander Hramov ${ }^{2,3}$, \\ Alexander Pisarchik ${ }^{4}$, Mikhail Lebedev ${ }^{5}$ \\ 1 Institute of Information Technologies, Mathematics and Mechanics, Lobachevsky \\ State University of Nizhny Novgorod, Nizhny Novgorod, Russia. \\ 2 Neuroscience and Cognitive Technology Laboratory, Innopolis University, The \\ Republic of Tatarstan, Russia. \\ 3 Saratov State Medical University, Saratov, Russia. \\ 4 Center for Biomedical Technology, Technical University of Madrid, Pozuelo de \\ Alarcón, Spain. \\ 5 Duke Center for Neuroengineering, Duke University, Durham, NC, United States \\ *maximenkovl@gmail.com
}

\begin{abstract}
Brain optimally utilizes resources to resist mental fatigue during the prolonged period of cognitive activity. Neural mechanisms underlying long-term cognitive performance remain unknown. We show that during the 40-minutes visual stimuli classification task, subjects improve behavioral performance in terms of response time and correctness. We observe that the prestimulus $\theta$ and $\alpha$ power grows during the experiment manifesting the mental fatigue. The prestimulus $\beta$ power, in its turn, increases locally in the region, engaged in the ongoing stimulus processing, that may reflect the neuronal adaptation. Our results evidence that the neuronal adaptation is enhanced in the course of the experiment reducing the cognitive demands required to activate the stimulus-related brain regions.
\end{abstract}

\section{Introduction}

Since the brain resource is limited, human exhibits mental fatigue during the prolonged periods of cognitive activity. The mental fatigue negatively correlates with human attention and results in the decrease of the behavioral performance 11. The bulk of literature describes the cortical activity underlying mental fatigue and attention. Thus, the mental fatigue is accompanied with the shift of EEG power toward the low-frequency bands, e.g. $\delta(1-4 \mathrm{~Hz}), \theta(4-8 \mathrm{~Hz})$, and $\alpha(8-13 \mathrm{~Hz})$ [2]3], while high-frequency activity, e.g. $\beta(15-30 \mathrm{~Hz})$ and $\gamma(>30 \mathrm{~Hz})$ typically decreases in amplitude 4 . Attention, in turn, is characterized by the low $\alpha$ - and high $\beta$-band spectral power. In particular, low $\alpha$ - and high $\beta$-band power during the prestimulus period reflects increased attention and predicts better performance in the ongoing task [5,6].

At the same time, cognitive performance is not necessary to decrease while accomplishing prolonged resource-demanding tasks. For instance, the increased reward makes the subject to improve behavioral performance even after the fatigue-induced session $7,8,8$. It evidences that the brain spends cognitive resources in the way to reserve it for future use 9 . The Ref. [10] relates the strategy the brain utilizes the 
cognitive resources with a fatigue-induced performance decrement, but not with the subjective fatigue. It manifests that the resource reallocation is controlled by the endogenous cognitive mechanisms regardless of the subjective feelings. According to the recent review [11, the neural activity underlying the cognitive resource rearrangement and cognitive performance is yet to be determined.

In this work, we subject the group of volunteers to the prolonged $(\sim 40 \mathrm{~min})$ task. The task requires the participants to quickly percept the successively presented bistable visual stimuli and report one of their possible interpretations right after the presentation. On the behavioral level, subjects improve their performance in the course of the task: they reduce their response time along with the number of errors. We suppose that this observation can be referred to as the training effect and suggest that within-experiment training is one of the possible mechanisms of the optimal cognitive resource utilization.

The previous works reported that the training in the particular task allowed the subjects to improve their behavioral performance. In the visual task, training improved the efficiency of high-level visual processing, which therefore provided less ambiguous sensory information to the decision-related brain networks [12. While in Ref. [12] the training period has lasted for three days; our results suggest that the training effect is notable even within a 40-min session.

Tang and Posner 13 previously described the brain's ability to enhance its cognitive performance in the course of training. They distinguished two different types of training - network training and state training [14]. The network training involves the practice of a specific task (e.g., attention, working memory) and thus exercises the specific task-related brain areas and networks 15]. The state training involves exercises, e.g., meditation that might alter the brain state in general 16. The network training, in its turn, may use a fixed task difficulty where performance shows improvement over trials or the adaptive training in which task difficulty adjusts as learning occurs [14]. Given the above, we suppose that our current study deals with the brain network training performed in the fixed difficulty fashion.

According to [14, the neuronal mechanisms underlying the training effect remain poorly understood. Some studies report the increased activation of the task-related brain networks in the course of training. In contrast, others observe the decrease of the task-related activity of the corresponding brain structures [17]. It also appears that the direction of the effect depends heavily on the type of training. For instance, the increased task-related brain activity may reflect the need for increased effort to solve more complicated tasks during adaptive training [18. Thus, there is no complete understanding of how brain activity changes during the training.

To address this issue, we consider the cortical activity on the EEG sensor level in $\theta$ (4-8 Hz), $\alpha(8-12 \mathrm{~Hz})$, and $\beta(15-30 \mathrm{~Hz})$ frequency bands in the course of the experiment. We observe that the training changes the brain activity not only during the stimulus processing stage but during the prestimulus period as well. The prestimulus $\theta$ and $\alpha$-band power grow in the course of the experiment that indicates the mental fatigue caused by the increased time spent performing the task. The prestimulus $\beta$-band power increases in the right hemisphere that may reflect the activation of the attention network. Finally, we show that the prestimulus $\beta$-band power increases locally in the region, engaged in the ongoing stimulus processing, that may reflect the neuronal adaptation [19]. We hypothesize that the neuronal adaptation is enhanced in the course of the experiment reducing the cognitive demands required to activate the stimulus-related brain regions. 


\section{Materials and methods}

\section{Participants}

Twenty healthy subjects (11 males and 9 females) aged from 26 to 35 with normal or corrected-to-normal visual acuity participated in the experiments. All of them provided written informed consent in advance. All participants were familiar with the experimental task and did not participate in similar experiments in the last six months. The experimental studies were performed under the Declaration of Helsinki and approved by the local Research Ethics Committee of the Innopolis University.

\section{Visual stimuli}

The ambiguous visual stimulus was the Necker cube 20,21. A subject without any perceptual abnormalities perceives the Necker cube as a 3D-object due to the specific position of the cube's edges. The Necker cube can be interpreted as left- or right-oriented depending on the contrast of the inner edges. The contrast of the three middle edges centered in the left middle corner was used as a control parameter $a \in[0,1]$. The values $a=1$ and $a=0$ correspond, respectively, to 0 (black) and 255 (white) pixels' luminance of the inner lines using the 8-bit gray-scale palette. Therefore, the control parameter was defined as $a=g / 255$, where $g$ is the brightness of the inner lines. In our experiment, we used the Necker cube images with eight different ambiguity levels (Fig. 1. A). Half of them $(a \in\{0.0,0.15,0.4,0.45\})$ are considered as left-oriented $(\mathrm{LO})$ and another half $(a \in\{0.55,0.6,0.85,1\})$ as right-oriented (RO). While for $a \approx 0$ and $a \approx 1$ (low ambiguous (LA) images) the cubes can easily be interpreted as left- and right-oriented, for $a \approx 0.5$ the identification of the cube orientation is a more difficult task since we deal with highly ambiguous (HA) images 22, 23]. The 14.2-cm Necker cubes were drawn by black and gray lines on a white background at the center of a 24 " BenQ LCD monitor with a spatial resolution of $1920 \times 1080$ pixels and a $60-\mathrm{Hz}$ refresh rate. The subjects located at a $70-80 \mathrm{~cm}$ distance from the monitor with a visual angle of approximately $0.25 \mathrm{rad}$.

\section{Experimental protocol}

The whole experiment lasted $\approx 40$ min for each participant, including short recordings of the resting EEG state $(\approx 150 \mathrm{~s})$ before and after the main part of the experiment. During experimental sessions, the Necker cubes with predefined values of $a$ (chosen from the set in Fig. 1. A) were randomly demonstrated 400 times, each cube with a particular ambiguity was presented about 50 times.

The scheme of the experimental session is shown in Fig. 1. B. Each $i$-th stimulus was presented for the time interval $\left(\tau_{i}\right)$, followed by the time interval $\left(\gamma_{i}\right)$ of the abstract image presentation (see Fig. 1. C). The participants were instructed to press the left or the right key with respectively left or right hand to report their interpretation of the orientation (left or right) of each cube. The consecutively presented images affect the perception of previously demonstrated cubes. For example, if the subject observed several left-oriented cubes in a row, then his/her perception was stabilized to the left-oriented cube, even if the next cube was right-oriented. Such phenomenon is referred to as a stabilization effect 24]. To reduce this effect, the duration of the stimulus exhibition varied in the range of $\tau \in[1,1.5] \mathrm{s}$. Moreover, a random variation of the control parameter $a$ also prevented the perception stabilization. Lastly, to draw away the observer's attention and make the perception of the next Necker cube independent of the previous one, different abstract pictures were exhibited for about $\gamma \in[3,5]$ s between subsequent demonstrations of the Necker cube images. For each 

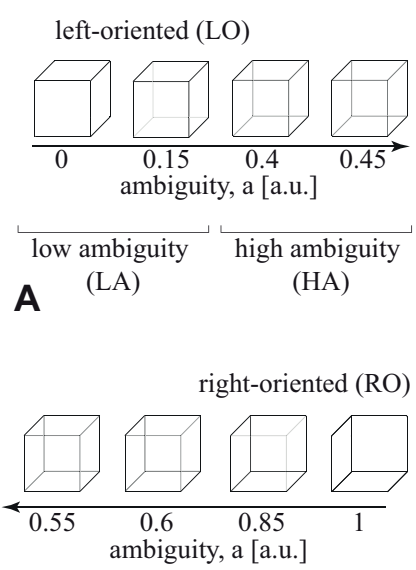

high ambiguity low ambiguity (HA)

(LA)

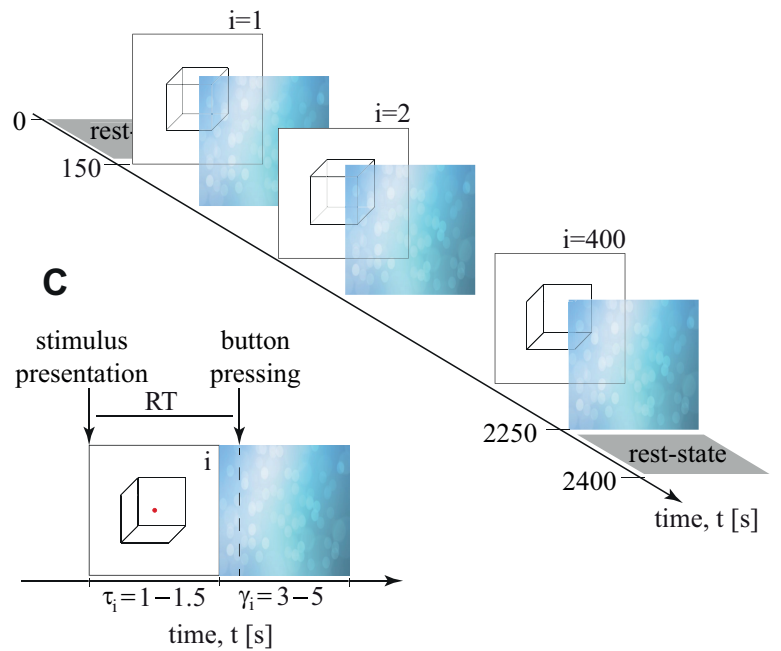

D

Fig 1. Visual stimuli The set of visual stimuli, Necker cubes, with different degree of ambiguity $a$ including high-ambiguity (HA) and low-ambiguity (LA) stimuli. A: left-oriented (LO) Necker cubes. B: right-oriented (RO) Necker cubes. C: Schematic illustration of experimental sessions. $\tau_{i}$ is the duration of the $i$-th cube presentation, $\gamma_{i}$ is the interval between the $i$-th and $(i+1)$-th presentations. D: Response time (RT) definition.

cube, we estimated a behavioral response by measuring the response time (RT), which corresponded to the time passed from the stimulus presentation to the button pressing (Fig. 1. D) and the error (if the subject's interpretation differs from the actual cube orientation).

\section{EEG acquisition and preprocessing}

We recorded the EEG signals using the monopolar registration method (a 10-10 system proposed by the American Electroencephalographic Society 25]). We recorded 31 signals with two reference electrodes A1 and A2 on the earlobes and a ground electrode $\mathrm{N}$ just above the forehead. We used the cup adhesive $\mathrm{Ag} / \mathrm{AgCl}$ electrodes placed on the "Tien-20" paste (Weaver and Company, Colorado, USA). Immediately before the experiments started, we performed all necessary procedures to increase skin conductivity and reduce its resistance using the abrasive "NuPrep" gel (Weaver and Company, Colorado, USA). Usually, the impedance varied within a $2-5 \mathrm{k} \Omega$ interval during the experiment. The electroencephalograph "Encephalan-EEG-19/26" (Medicom MTD company, Taganrog, Russian Federation) with multiple EEG channels and a two-button input device (keypad) performed amplification and analog-to-digital conversion of the EEG signals. This device possessed the registration certificate of the Federal Service for Supervision in Health Care No. FCP 2007/00124 of 07.11.2014 and the European Certificate CE 538571 of the British Standards Institute (BSI). The raw EEG signals were sampled at $250 \mathrm{~Hz}$, filtered by a band-pass FIR filter with cut-off points at $1 \mathrm{~Hz}$ (HP) and $100 \mathrm{~Hz}$ (LP) and by a 50-Hz notch filter by embedded a hardware-software data acquisition complex. Eyes blinking and heartbeat artifact removal was performed by Independent Component Analysis (ICA) using EEGLAB software 26. The recorded EEG signals presented in proper physical units (millivolts) were segmented into two sets of 4-s trials, including 2-s prestimulus (baseline) activity and 2-s poststimulus activity. Data were then inspected manually and corrected for remaining artifacts. 


\section{Trials selection and experimental conditions}

After the EEG preprocessing procedure, we excluded some trials due to high-amplitude artifacts. To keep the number of EEG trials constant for each cube ambiguity, we considered 320 trials out of the initial 400, including 40 trials for each ambiguity. To define the experimental conditions, we divided the whole experimental session into six non-overlapping fragments $\left(T_{i}\right)$ of the equal length. For each fragment, we selected 40 trials with an equal proportion of RO and LO stimuli, as well as LA and HA stimuli. Such a selection of the trials enables neglecting the effects caused by the cube orientation and complexity. For each segment, we averaged RT across the 20 trials corresponding to LA and HA stimuli and compared the mean RT values across the conditions $T_{1} \ldots T_{6}$ in the group of subjects.

\section{EEG time-frequency analysis}

Time-frequency analysis of EEG trials was carried out using Morlet wavelet with the number of cycles for each frequency $f$ as $f / 2$. The wavelet power $E$ was calculated in the $4-30 \mathrm{~Hz}$ frequency band and averaged over the frequency bands of interest: $\theta$ $(4-8 \mathrm{~Hz}), \alpha(8-12 \mathrm{~Hz})$ and $\beta(15-30 \mathrm{~Hz})$. The prestimulus oscillatory activity was averaged over the time interval from $-0.5 \mathrm{~s}$ to $0 \mathrm{~s}$. The poststimulus oscillatory activity was contrasted by the activity in the prestimulus period subtracting the mean of baseline values followed by dividing by the mean of baseline values ('percent' mode) and considered in the time interval from $0 \mathrm{~s}$ to $0.5 \mathrm{~s}$. The results of the time-frequency analysis were averaged over trials for each subject belonging to each fragment $\left(T_{1}\right.$ $\left.\ldots T_{6}\right)$.

Following the Ref. 27], a group-level spatio-temporal non-parametric cluster-based statistical test was used to address the statistical significance of the $\theta, \alpha$ and $\beta$ oscillatory activity changes in the prestimulus and poststimulus states between the different conditions $T_{i}$. Here, a pairwise comparison of samples between the conditions was performed via paired sample t-test with a critical level of $p_{\text {pairwise }}$ set to 0.01 . The critical level for non-parametric cluster-based statistical test $p_{\text {cluster }}$ was set to 0.05 . The number of random permutations was 2000. The time-frequency analysis and non-parameteric cluster-based statistical tests were performed using MNE package for Python 3.7 [28]. To estimate the inter-hemispherical asymmetry of prestimulus oscillatory activity in the $\theta, \alpha$ and $\beta$ bands between the conditions $T_{i}$, the EEG spectral power of the corresponding frequency bands was averaged over the EEG sensors placed in the left hemisphere (LH) \{O1, P3, T5, CP3, TP7, C3 T3, FC3 FT7, F3, F7, FP1 and the right hemisphere $(\mathrm{RH})\{\mathrm{O} 2, \mathrm{P} 4, \mathrm{~T} 6, \mathrm{CP} 4, \mathrm{TP} 8, \mathrm{C} 4, \mathrm{~T} 4, \mathrm{FC} 4, \mathrm{FT} 8, \mathrm{~F} 4, \mathrm{~F} 8$, FP2\}.

\section{Results}

Behavioral results. We compared RT across the fragments $T_{i}$ via a repeated-measures ANOVA with two factors: ambiguity (LA and HA) and fragment $\left(T_{1} \ldots T_{6}\right)$. As a result, ANOVA with the Greenhouse Geisser correction revealed the significant main effect for fragment $\left(F_{2.31,44.05}=9.63, p<0.001\right)$ and ambiguity $\left(F_{1,19}=59.66, p<0.001\right)$, whereas the interaction effect fragment $\times$ ambiguity was insignificant $\left(F_{2.47,46.93}=1.06, p=0.366\right)$. We concluded that RT changed between the fragments similarly regardless of the stimulus ambiguity. Therefore, for further analysis, we combined LA and HA stimuli on each fragment. The post-doc analysis via the Bonferroni-corrected paired-samples $t$-test revealed a monotonous decrease of RT across the fragments $T_{1} \ldots T_{6}$ (Fig. 2, A) with the maximal statistically significant difference 
achieved between the beginning $\left(T_{1}\right)$ and the end $\left(T_{6}\right)$ of the experiment $(\mathrm{M}=0.189$, $\mathrm{SE}=0.043, t(19)=4.318, p=0.006)$. The detailed analysis of pairwise differences revealed that 18/20 subjects shown an effect in the same direction as the group (Fig. 2 B).

A similar tendency was observed for the error rate (ER) (Fig. 2, C). ANOVA with the Greenhouse Geisser correction revealed a significant effect for the fragment $\left(F_{2.75,52.38}=3.386, p=0.028\right)$. The posthoc analysis with the paired samples $t$-test displayed the maximal statistically significant difference between $T_{1}$ and $T_{6}(\mathrm{M}=1.74$, $\mathrm{SE}=0.64, t(19)=2.733, p=0.013)$. The Bonferroni-corrected $p$-value was 0.198. The distribution of pairwise differences reflected that $11 / 20$ subjects followed the group tendency and 5/20 subjects shown no effect (Fig. 2, D).

The obtained results demonstrated that both the RT and the ER decreased in the course of the experiment. We supposed that the most significant difference in the neuronal activity should be observed between the fragments $T_{1}$ and $T_{6}$. For these conditions, we compared RT between the $\mathrm{LO}$ and RO cubes. The repeated-measures ANOVA with the Greenhouse Geisser correction revealed a significant change in RT between $T_{1}$ and $T_{6}\left(F_{1,19}=20.714, p<0.001\right)$. In contrast, RT changed insignificantly between LO and RO cubes $\left(F_{1,19}=1.56, p=0.227\right)$. Finally, we found that the interaction effect was also insignificant $\left(F_{1,19}=0.084, p=0.775\right)$.

This analysis evidenced that a change in RT between the $T_{1}$ and $T_{6}$ conditions was independent of the cube ambiguity and orientation. Given above, cortical activity was compared between $T_{1}$ and $T_{6}$ conditions based on 40 trials per condition, including the equal proportion of the LA and HA stimuli as well as LO and RO stimuli.

Rest-state and prestimulus activity. First, we analyzed how the subject's condition changed in the course of the experiment. The human condition affects brain activity regardless of the presented stimuli or the task. Therefore, we analyzed the EEG spectral power in three conditions: rest-state (the phase of the experiment before the first stimulus was presented), prestimulus state at the beginning of the experiment $\left(T_{1}\right)$ and prestimulus state at the end of the experiment $\left(T_{6}\right)$. For each condition, we considered the set of 40 EEG trials (0.5-s length). Fig. 3 illustrates the EEG spectral power in the $\theta, \alpha$, and $\beta$ bands in the left (LH) and right hemispheres (RH) for these conditions.

In the $\theta$-band (Fig. 3, A) the repeated measures ANOVA with the Greenhouse-Geisser correction revealed insignificant main effect for the hemisphery $\left(F_{1.00,19.00}=0.18, p=0.676\right)$ and for the condition $\left(F_{1.058,20.096}=0.18, p=0.124\right)$. The interaction effect hemisphere $\times$ condition was also insignificant

$\left(F_{1.067,20.268}=0.823, p=0.383\right)$.

In the $\alpha$-band (Fig. 3, B), the repeated measures ANOVA with the Greenhouse-Geisser correction revealed a significant main effect for the condition $\left(F_{1.46,27.84}=9.713, p=0.002\right)$ and the hemisphere $\left(F_{1.00,19.00}=4.944, p=0.039\right)$. The interaction effect condition $\times$ hemisphere was insignificant

$\left(F_{1.27,24.146}=1.378, p=0.264\right)$. The post-hoc analysis with Bonferroni-corrected paired samples $t$-test demonstrated that $E_{\alpha}(\mathrm{RH})$ exceeded $E_{\alpha}(\mathrm{LH})\left(\mathrm{M}=9.06 \times 10^{3}\right.$,

$\mathrm{SE}=4.07 \times 10^{3}, p=0.039$ ). The distribution of the pairwize differences (Fig. 3 . B) evidenced that $14 / 20$ subjects followed the group tendency at the rest-state, while $12 / 20$ and 14/20 subjects followed the group tendency at $T_{1}$ and $T_{6}$ conditions, respectively.

$E_{\alpha}$ in the rest-state did not differ significantly from $E_{\alpha}\left(T_{1}\right)(p=1.0)$, while $E_{\alpha}\left(T_{6}\right)$ significantly exceeded both $E_{\alpha}\left(T_{1}\right)\left(\mathrm{M}=15.9 \times 10^{3}, \mathrm{SE}=4.84 \times 10^{3}, p=0.012\right)$ and $E_{\alpha}$ during the rest-state $\left(\mathrm{M}=12.8 \times 10^{3}, \mathrm{SE}=3.01 \times 10^{3}, p=0.001\right)$. The pairwize diffeences evidenced that $15 / 20$ and $18 / 12$ subjects followed the group effect when $T_{6}$ was compared to the rest-state and $T_{1}$ conditions.

We concluded that participants exhibited the right-lateralization of the $\alpha$-band 

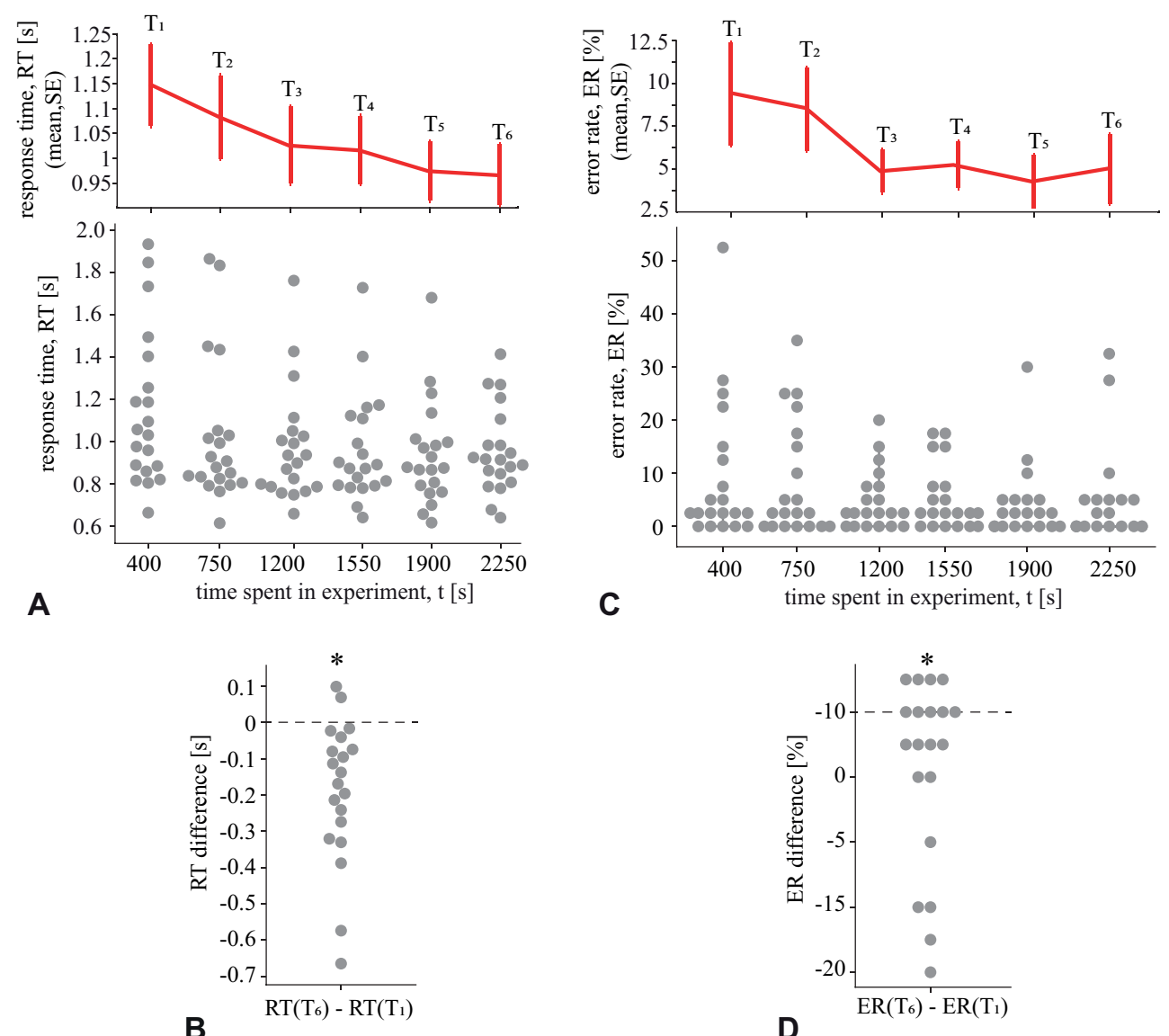

Fig 2. Behavioral performance in the course of the experiment A: The response time (RT) for each fragment $\left(T_{1} \ldots T_{6}\right)$ of the experimental session. The upper panel demonstrates the group mean $\pm \mathrm{SE}$, while the lower panel reflects the data of all subjects. B: The error rate (ER) for each fragment of the experimental session (mean $\pm \mathrm{SE}$ and the individual values). C: The change of $\mathrm{RT}$ between the last $\left(T_{6}\right)$ and the first $\left(T_{1}\right)$ fragments. D: The change of ER between these fragments.

power during the rest-state and in the prestimulus state during the experiment. The overall prestimulus $\alpha$-band power at the beginning of the experiment did not differ from the rest-state. The overall $\alpha$-band power in the prestimulus period increased in the course of the experiment.

In the $\beta$-band (Fig. 3 C), repeated measures ANOVA with Greenhouse-Geisser correction revealed a significant main effect for the condition $\left(F_{1.531,29.094}=4.654, p=0.025\right)$, and insignificant main effect for the hemisphere $\left(F_{1.00,19.00}=0.0, p=0.988\right)$. The interaction effect condition $\times$ hemisphere was also significant $\left(F_{2,38}=7.619, p=0.002\right)$. The post-hoc analysis with Bonferroni-corrected paired samples $t$-test demonstrated that $E_{\beta}$ in the rest-state did not differ significantly from $E_{\beta}\left(T_{1}\right)(p=1.0)$, and $E_{\beta}\left(T_{6}\right)$ did not significantly differ from both $E_{\beta}\left(T_{1}\right)$ $(p=0.136)$ and $E_{\beta}$ during the rest-state $(p=0.066)$. At the same time, analysis of the pairwize differences (Fig. 3. C) revealed that $16 / 20$ and $18 / 20$ subjects exhibited higher $E_{\beta}\left(T_{6}\right)$ when compared to the $E_{\beta}$ at the rest-state and to the $E_{\beta}\left(T_{1}\right)$, respectively.

The paired samples $t$-test revealed that $E_{\beta}(\mathrm{LH})$ and $E_{\beta}(\mathrm{RH})$ differed neither for the rest-state $(t(19)=0.423, p=0.677)$ nor for the prestimulus period at the beginning $(t(19)=1.49, p=0.151)$ and at the end $(t(19)=-1.62, p=0.121)$ of the experiment. 

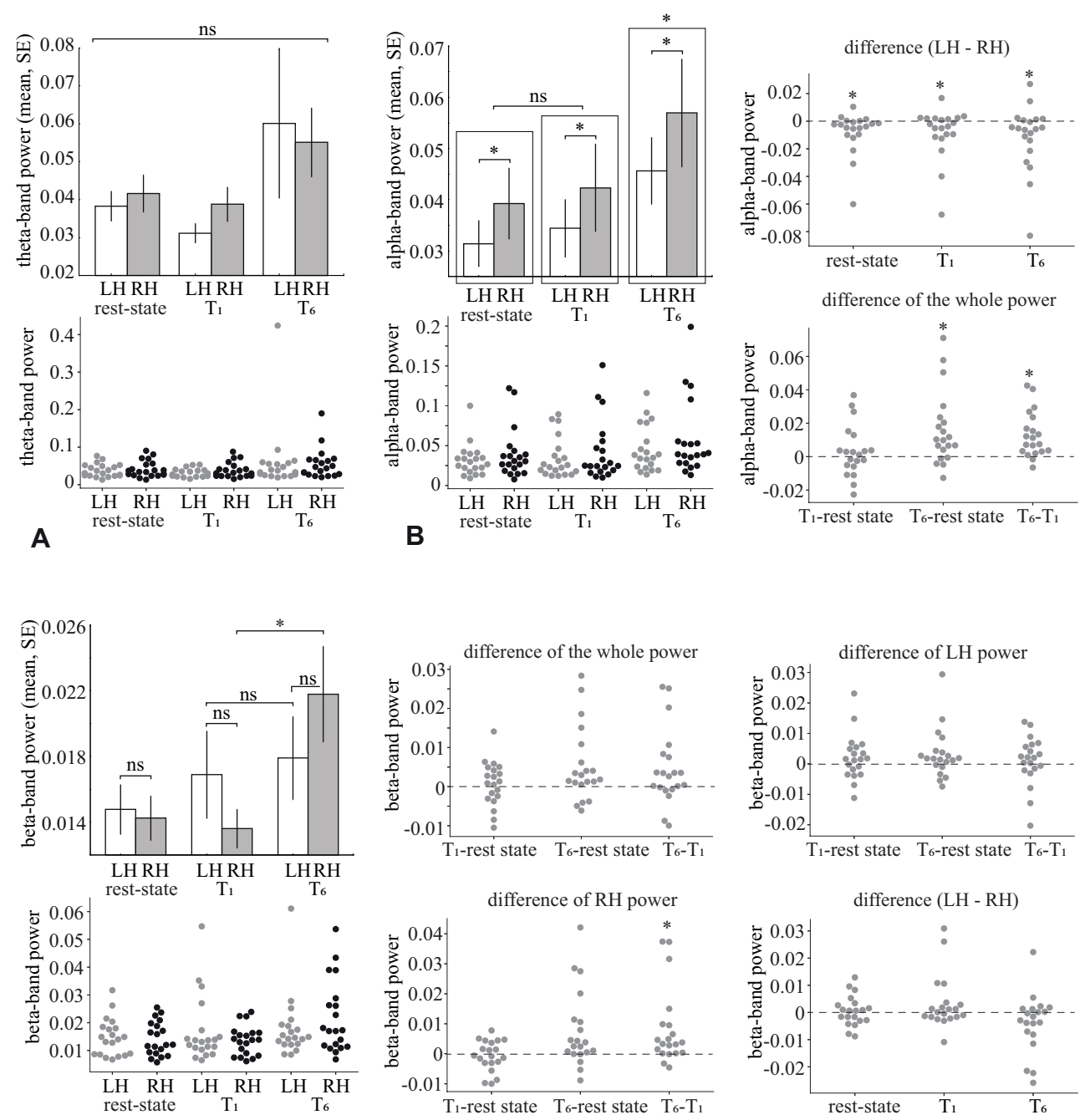

\section{C}

Fig 3. Rest-state and prestimulus wavelet power A: $\theta$-band power in the left hemisphere $(\mathrm{LH})$ and the right hemisphere $(\mathrm{RH})$ during three conditions (rest-state, prestimulus period at the beginning $\left(T_{1}\right)$ and prestimulus period at the end $\left(T_{6}\right)$ of the experiment). The upper panel represents group mean $\pm \mathrm{SE}$, and the lower panel shows the data of all subjects. B: $\alpha$-band power across the same conditions (mean $\pm \mathrm{SE}$ and the individual values). Differences between the $\mathrm{LH}$ and $\mathrm{RH} \alpha$-band power for the rest-state, $T_{1}$ and $T_{6}$ conditions. The differences of the $\alpha$-band power in both hemispheres between the different conditions. $\mathrm{C}$ : $\beta$-band power in the left hemisphere $(\mathrm{LH})$ and the right hemisphere $(\mathrm{RH})$ for the rest-state, $T_{1}$ and $T_{6}$ conditions (mean $\pm \mathrm{SE}$ and the individual values). The differences of the $\beta$-band power in both hemispheres between the different conditions. The differences of the $\mathrm{RH} \beta$-band power between the different conditions. The differences of the LH $\beta$-band power between the different conditions. Differences between the $\mathrm{LH}$ and $\mathrm{RH} \beta$-band power for the rest-state, $T_{1}$ and $T_{6}$ conditions.

A paired-samples $t$-test was conducted to compare the $\beta$-band power in the right hemisphere $E_{\beta}(\mathrm{RH})$ across the conditions. We revealed that $E_{\beta}(\mathrm{RH})$ at $T_{1}$ did not differ from the $E_{\beta}(\mathrm{RH})$ at the rest-state $(t(19)=0.562, p=0.58) . E_{\beta}(\mathrm{RH})$ at $T_{6}$ significantly exceeded $E_{\beta}(\mathrm{RH})$ at $T_{1}\left(\mathrm{M}=8.17 \times 10^{3}\right.$, 


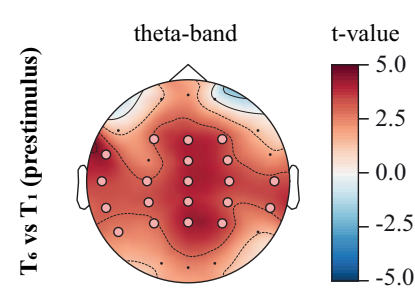

A

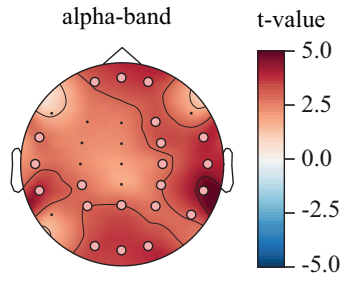

B

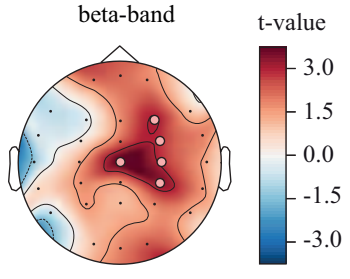

C

Fig 4. Prestimulus wavelet power distribution The $t$-value and the EEG channel clusters representing significant changes in (A) $\theta-$, (B) $\alpha$ - and (C) $\beta$-frequency band spectral power at the end $\left(T_{6}\right)$ versus the beginning $\left(T_{1}\right)$ of the experiment $\left(p_{\text {pairwise }}<0.01, p_{\text {cluster }}<0.05\right)$

$\left.\mathrm{SE}=2.81 \times 10^{3}, t(19)=2.899, p=0.009\right)$. Analysis of the pairwize differences (Fig. 3. C) revealed that $18 / 20$ subjects shown this effect. The difference of $E_{\beta}(\mathrm{RH})$ between $T_{6}$ and rest-state was also significant $\left(\mathrm{M}=7.53 \times 10^{3}\right.$, $\left.\mathrm{SE}=2.85 \times 10^{3}, t(19)=2.639, p=0.016\right)$. This effect was observed for $17 / 20$ subjects.

A paired-samples $t$-test was conducted to compare the $\beta$-band power in the left hemisphere, $E_{\beta}(\mathrm{LH})$, in the rest-state, $T_{1}$ and $T_{6}$ conditions. We revealed that $E_{\beta}(\mathrm{LH})$ at $T_{1}$ did not differ from the $E_{\beta}(\mathrm{LH})$ at the rest-state $(t(19)=1.273, p=0.218)$. $E_{\beta}(\mathrm{LH})$ at $T_{6}$ did not differ from both $E_{\beta}(\mathrm{LH})$ at $T_{1}(t(19)=0.565, p=0.579)$ and at the rest-state $(t(19)=1.733, p=0.099)$.

Finally, according to the paired-samples $t$-test, $E_{\beta}(\mathrm{RH})$ did not significantly differ from $E_{\beta}(\mathrm{LH})$ at the rest-state $(t(19)=-0.423, p=0.677)$ as well as at $T_{1}$ $(t(19)=-1.495, p=0.151)$ and $T_{6}(t(19)=1.624, p=0.121)$ conditions.

The obtained results demonstrated that the $\beta$-band power did not change at the beginning of the experiment when compared to the rest-state. In the course of the experiment, we observed an increase in the prestimulus $\beta$-band spectral power in the right hemisphere.

Fig. 4 shows the topograms of $t$-values reflecting the power changes in the $\theta-, \alpha-$, and $\beta$-frequency bands in the $T_{6}$ versus $T_{1}$ conditions.

In the $\theta$-band (Fig. 4, A), the cluster-based statistical analysis with permutations revealed that the prestimulus state at the end of the experiment is characterized by the increasing power over the EEG channel cluster in the parietal, sensorimotor, frontal and temporal areas.

In the $\alpha$-band (Fig. 4, B), a significant increase of $\alpha$-band power at the end of the experiment was observed for the channel clusters in the occipito-parietal area, frontal area, and temporal areas.

In the $\beta$-band (Fig. $4, \mathrm{C}$ ), the prestimulus state at the end of the experiment was characterized by a significant $(p<0.01)$ increase in the $\beta$-band spectral power for the right-lateralized channel cluster including channels F4, FC4, C4, CP4, Cz.

Task-related activity. The task-related cortical activity on the EEG sensor level was analyzed by comparing the topograms of the $\theta-, \alpha$ - and $\beta$-band spectral power between the beginning $\left(T_{1}\right)$ and the end $\left(T_{6}\right)$ of the experiment. The Fig. 5 illustrates the channels clusters (circles) and the corresponding $t$-values (color scale) obtained during the 0.5-s interval following the presentation of the visual stimulus. Each topogram represented the spectral power averaged over a $0.1-\mathrm{s}$ window mentioned in the legend under the topograms.

One could see that the clusters had different spatio-temporal properties in the different frequency bands. At the same time, no positive clusters appeared in these bands, indicating the overall increase of the task-related neuronal activation at the 
$T_{6}$ vs $T_{1}$ (task-related)

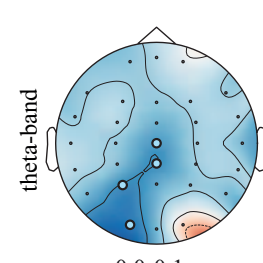

$0.0-0.1 \mathrm{~s}$

A

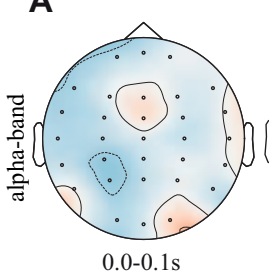

$0.0-0.1 \mathrm{~s}$

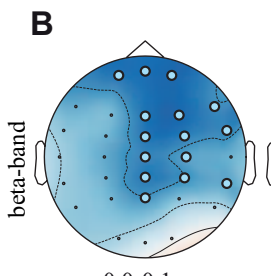

$0.0-0.1 \mathrm{~s}$

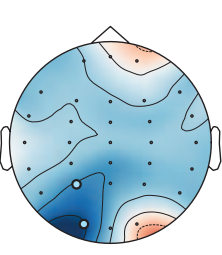

$0.1-0.2 \mathrm{~s}$

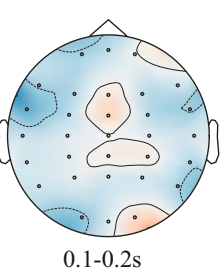

$0.1-0.2 \mathrm{~s}$

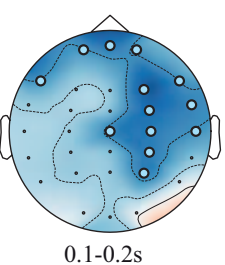

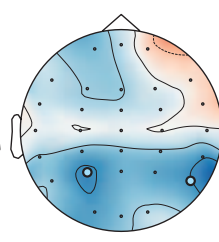

$0.2-0.3 \mathrm{~s}$
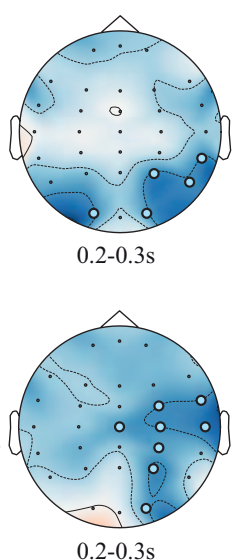

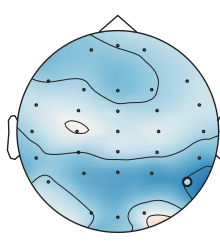

$0.3-0.4 \mathrm{~s}$
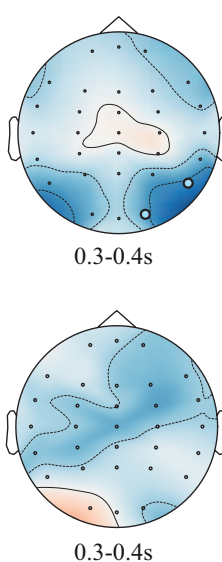
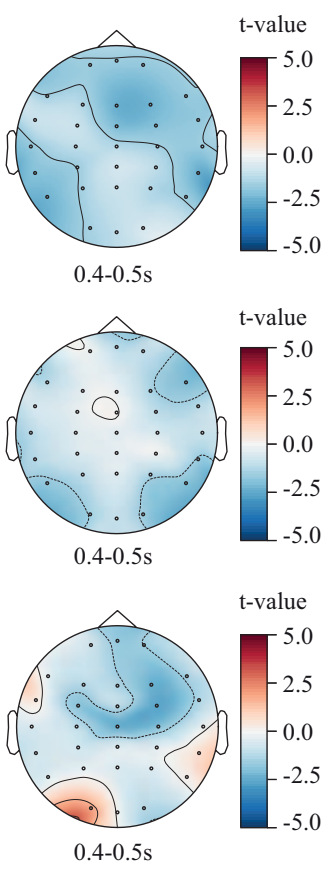

\section{C}

Fig 5. Task-related spatio-temporal activity. The $t$-value and the EEG channel clusters representing significant changes in the wavelet power in the (A) $\theta-$, (B) $\alpha$ - and (C) $\beta$-frequency bands at the end $\left(T_{6}\right)$ versus the beginning $\left(T_{1}\right)$ of the experiment $\left(p_{\text {pairwise }}<0.01, p_{\text {cluster }}<0.05\right)$

beginning of the experiment.

In the $\theta$-band (Fig. 5 a), the earlier processing stage $(t<0.1 \mathrm{~s})$ was characterized by the left-lateralized occipito-parietal cluster (O1 and P3 channels) and the mid-line centro-parietal cluster $(\mathrm{CPz}$ and $\mathrm{Cz}$ channels). For $(0.1<t<0.2 \mathrm{~s})$, the only left-lateralized occipito-parietal (O1 and P3) cluster was observed. The further processing $(t>0.2 \mathrm{~s})$ was associated with the increased $t$-value over the parietal and temporal EEG sensors. For $(0.2<t<0.3 \mathrm{~s})$, there were two clusters (P3 and P8), while for $(0.3<t<0.4 \mathrm{~s})$, the only one cluster $(\mathrm{P} 8)$ remained. Finally, for $(t>0.4 \mathrm{~s})$, no clusters were found in the $\theta$-frequency band.

The $\alpha$-band power (Fig. 5. b) remained unchanged during the first 0.2 sec after the stimulus presentation. Then, for $0.2-0.3 \mathrm{~s}$, we observed the bilateral negative cluster in the occipital area, including O1 and O2 EEG channels and the right-lateralized parieto-temporal cluster, including P4, P8, and TP8 EEG channels. For the $0.3-0.4 \mathrm{~s}$ the size of the observed cluster decreased, and it became right-lateralized. Finally, the further increase of the processing time led to the cluster disappearance. The obtained results evidenced that at the end of the experiment, the task-related cortical activity was characterized by the increased $\alpha$-band power in the occipital and right temporal areas during $0.2-0.3 \mathrm{~s}$ after the stimulus presentation.

In the $\beta$-frequency band (Fig. 5 , c), the negative cluster was observed for the first $0.3 \mathrm{~s}$ after the visual stimulus presentation. At the earlier sensory processing stage $(t<0.1 \mathrm{~s})$ it included the bilateral prefrontal cortical channels (Fp1, Fpz, Fp2), the right-lateralized frontoparietal area $(\mathrm{Fz}, \mathrm{F} 4, \mathrm{~F} 8, \mathrm{FCz}, \mathrm{FC} 4, \mathrm{Cz}, \mathrm{C} 4, \mathrm{CPz}, \mathrm{CP} 4, \mathrm{Pz})$ and the right temporal area (FT8, TP8). During the $0.1-0.2 \mathrm{~s}$, the observed cluster was bilateral in the prefrontal cortex, but right-lateralized in the temporal region. For 
$0.2-0.3 \mathrm{~s}$, the frontal cluster disappeared, and one could observe the remaining right-lateralized cluster in the occipital, parietal, and temporal cortex. Finally, no clusters were found for $t>0.3 \mathrm{~s}$ in the $\beta$-frequency band.

\section{Discussion}

Our results demonstrate that during a prolonged visual stimuli classification task, subjects enhance their performance in terms of the reduced reaction time and decision accuracy. This observation is not trivial since a prolonged cognitive activity may induce mental fatigue and, therefore, cognitive decline. Thus, we suppose that the brain implements a strategy for the optimal utilization of cognitive resources to resist mental fatigue and increase behavioral performance.

At the beginning of the experiment, we observe enhanced spectral power in the $\theta, \alpha$, and $\beta$ frequency bands during the stimulus processing. The high $\beta$ power has been observed in the right temporoparietal cortex and bilaterally in the prefrontal cortex, while high $\alpha$ and $\theta$ power - in the occipital and in both occipital and parietal areas, respectively.

The neuronal activity in the temporoparietal regions subserves the processing and storage of visuospatial information 29 30. At the same time, the activation of the prefrontal cortex indicates that the task accomplishing requires additional cognitive resources. It is also confirmed by an increase of the task-related $\theta$-band activity [31. The task-related occipital $\alpha$-power is associated with selective attention during stimulus processing. The low $\alpha$-band power reflects the effective processing of attended stimuli 32 . Having summarized, we suppose that at the beginning of the experiment, stimulus processing engages the resources of the frontoparietal cortical network.

Moreover, the lower task-related $\alpha$-band power in the occipital area at the end of the experiment is possible to reflect the enhanced brain ability to respond to the attended stimulus effectively.

The prestimulus activity in the $\theta, \alpha$, and $\beta$-bands changes in the course of the experiment. The prestimulus $\alpha$-band power is higher in the right hemisphere during the rest-state and the prestimulus period. This lateralization doest not change in the course of the experiment. At the same time, $\alpha$-band power grows during the experiment over the majority of the EEG sensors in both hemispheres. The $\theta$ - and $\beta$-band activity is bilateral in the rest-state and prestimulus period. However, at the end of the experiment, the $\beta$-band power increases in the right hemisphere, while the $\theta$-band power grows bilaterally in the frontal-central, parietal, and temporal regions.

The prestimulus activity in the $\alpha$ and $\beta$ bands is usually analyzed to characterize the subject's attention. A wide body of literature shows that $\alpha$ and $\beta$-band activities are relevant to attention in general and not restricted to the visual stimuli processing [6, 33 35]. Attention modulates the prestimulus $\alpha$ - and $\beta$-band power $6,33,36]$ and affects the stimulus processing accuracy. Thus, low $\alpha$ - and high $\beta$-band power during the prestimulus period is beneficial for sensory perception [5, 6]. The prestimulus $\theta$ - and $\alpha$-band activities can serve as the markers of mental fatigue, i.e., the increased power in these bands reflects the subject's mental fatigue and causes the performance decrement 3 . Taken together, the low prestimulus $\theta$ - and $\alpha$-band power and the high $\beta$-power should predict the high performance and vice versa. On the contrary, we report that the high prestimulus spectral power in the $\theta-, \alpha$ - and $\beta$ frequency bands accompany the performance increment.

According to [37, the lateralized $\alpha$-band power reflects the attentional breadth during the stimulus processing. The attentional breadth characterizes the subject's ability to focus attention either on the global level (e.g., the forest) or local elements that make up the stimulus (e.g., the trees) 38]. Recent studies demonstrate that greater
323

324

325

326 

over the right and left frontal-central sensors correlate with attentional breadth. The greater left frontal $\alpha$ power corresponds to faster responses to local targets and greater right frontal $\alpha$ power - to faster responses to global targets [40]. Previous findings suggest that prestimulus and rest-state $\alpha$ power lateralization [41] also predict the subject's performance in a global-local attention task 37].

We report the high $\alpha$-band activity in the right hemisphere at rest as well as in the prestimulus state. Moreover, the lateralization does not change in the course of the experiment. Therefore, we can conclude that the subjects which initially have right-lateralized alpha-band power exhibit the global attentional bias and do not change it during the experiment. It coincides with the existing literature claiming that the attentional bias remains stable for at least ten days in multiple global/local tasks 42 .

The increased prestimulus $\alpha$ power in the course of the experiment can be an electrophysiological indicator of mental fatigue 43. The prolonged task induces mental fatigue, increasing reaction times, misses, and false alarms. As discussed above, high $\alpha$-band power predicts the low performance of the ongoing stimulus processing. Thus, it can be supposed, that increased $\alpha$-band power at the end of the experiment is associated with mental fatigue, which should lead to the performance decline. The increased $\theta$-band power, together with $\alpha$-band power, can also manifest the mental fatigue. According to [3], the prestimulus $\theta$-band power in the frontal area grows in the course of the experiment and negatively correlates with the behavioral performance. Another paper [44], relates mental fatigue with the increased $\theta$-band power in the frontal-central and parietal regions.

High prestimulus $\beta$-band power in the right hemisphere can be related to the increasing human attention. There is a view that the human attentional brain network is overall lateralized to the right hemisphere 45]. Some components of the attention (e.g., alerting and disengaging functions) are bilateral [46], while others (e.g., orienting and executive functions) are biased to the right hemisphere [47]. According to [48, the executive functions subserve an interplay between alerting and orienting functions to maintain the state of readiness and focus attention towards the relevant features of a stimulus.

Taken together, the increased orienting and executive components of attention in the course of the experiment can be the possible reasons for the increase in behavioral performance. In this context, the performance can grow due to the enhanced effectiveness of the relevant stimulus features selection. At the same time, high prestimulus $\theta$ - and $\alpha$ - power contradict the definition of attentional state in general. Therefore, we cannot conclude unequivocally that the brain increases its attentional properties in the course of the experiment.

Finally, we introduce one more possible explanation for the increased performance in the course of the experiment. We observe that the prestimulus $\beta$-band power at the end of the experiment increases locally in the region, which is more engaged during the stimulus processing. Hence, we suppose that the preactivation of the stimulus-related cortical areas reduces the cognitive demands required for their activation during the stimulus processing ( $[49]$ ). The preactivation of the stimulus-related neuronal ensembles before the stimulus processing is also known as the neuronal adaptation (NA) The NA is considered as the evidence for the predictive coding theory 50. NA is observed when the same visual stimulus is repeatedly presented with a brief interval and causes the reduced neural response to repeated compared with unrepeated stimuli 19]. The NA is supposed to arise from at least two types of neural activity. One explanation is that only the part of the neuronal ensemble is sensitive to stimulus recognition. Thus, the neurons that are not critical for recognizing the stimulus decrease their responses as 
the stimulus is repeatedly presented. In contrast, the neuronal populations carrying essential information continue to give a robust response. As a result, the mean firing rate becomes attenuated by stimulus repetition. This theory is supported by the first studies of unit cell recordings 51. The alternative explanation is the stimulus repetition reduces the response in the temporal domain [19]. In this theory, the neural processing network settles to a stable response more quickly in response to a repeated than novel stimulus, because the network connections involved in producing the response have been reinforced by a previous presentation of the same stimulus [52]. The NA affects the neuronal response in the occipital [53], parietal [54], and frontal [55] cortical populations in the single-unit data as well as on the sensor level.

Taking together, we suppose that the training effect in the course of the experiment results in the preactivation of the stimulus-related regions in the $\beta$ frequency band. The very recent work [56] also relates the $\beta$-band spectral power to the training. The authors report on the enhanced $\beta$-band power over the central, temporal and the parietal sensors during an $80-400 \mathrm{~ms}$ window of the poststimulus onset (post- versus pre-training trials), while we observe that the $\beta$-band spectral power decreases in the same areas during the same time window. At first glance, this issue can be addressed to different experimental paradigms. For example, [56] compared two experiments carried out in different days, whereas in this work, we analyze a single experimental session performed in one day. It follows that the different mechanisms stay behind the training effect in these two paradigms. Besides, different stimuli are used, i.e., we consider visual stimuli only, while [56] uses visual stimuli together with auditory stimuli. Moreover, similar to our findings 56] report on a significant decrease in the $\beta$-band power (post-training vs. pre-training trials) at certain conditions. The authors relate this effect to the participant's perceptual template formation after the training and consider these results as the evidence for the predictive coding model. The predictive coding theory suggests that the brain creates sensory templates [57, which causes increasing $\beta$-band activity before the occurrence of an expected event that potentially reflects the mobilization of neuronal populations to encode the expected sensory inputs 58,59 .

\section{Supporting information}

S1 Data. EEG and behavioral data. This structure contains 20 cells representing data of 20 subjects. Each cell includes field Trial, which contains 80 EEG trials recorded for 31 channels. The field Label contains the names of the channels. The first 40 trials are chosen from the beginning of the experiment and belong to the condition $T_{1}$. The rest 40 trials are chosen from the end of the experiment and belong to the condition $T_{6}$. Each trial has $4 \mathrm{~s}$ length, and the stimulus is presented in the middle of the trial. The field PresentationMoment contains the moments of the stimuli presentations and Ambiguity contains the stimulus ambiguity value. The field Reaction Time contains the reaction time for each trial, and the field Button contains the identification of the button pressed (1-for the left and 2-for the right). The field Background contains the rest-state EEG signals recorded before the stimuli presentation. EEG signals are sampled at $250 \mathrm{~Hz}$, filtered by a band-pass FIR filter with cut-off points at $1 \mathrm{~Hz}(\mathrm{HP})$ and $100 \mathrm{~Hz}(\mathrm{LP})$ and by a 50-Hz notch filter. Eyes blinking and heartbeat artifact are removed with Independent Component Analysis (ICA)

\section{Acknowledgments}

This work has been supported by the Russian Foundation for Basic Research (Grant 19-32-60042) in the part of experimental work and behavioral data analysis. AK is 
supported by the President Program (MK-1760.2020.2) in the part of experimental data preprocessing.

\section{References}

1. Mizuno K, Tanaka M, Yamaguti K, Kajimoto O, Kuratsune H, Watanabe Y. Mental fatigue caused by prolonged cognitive load associated with sympathetic hyperactivity. Behavioral and brain functions. 2011;7(1):17.

2. Phipps-Nelson J, Redman JR, Rajaratnam SM. Temporal profile of prolonged, night-time driving performance: breaks from driving temporarily reduce time-on-task fatigue but not sleepiness. Journal of sleep research. 2011;20(3):404-415.

3. Wascher E, Rasch B, Sänger J, Hoffmann S, Schneider D, Rinkenauer G, et al. Frontal theta activity reflects distinct aspects of mental fatigue. Biological psychology. 2014;96:57-65.

4. Lal SK, Craig A. Electroencephalography activity associated with driver fatigue: Implications for a fatigue countermeasure device. Journal of Psychophysiology. 2001;15(3):183.

5. Van Dijk H, Schoffelen JM, Oostenveld R, Jensen O. Prestimulus oscillatory activity in the alpha band predicts visual discrimination ability. Journal of Neuroscience. 2008;28(8):1816-1823.

6. Gola M, Magnuski M, Szumska I, Wróbel A. EEG beta band activity is related to attention and attentional deficits in the visual performance of elderly subjects. International Journal of Psychophysiology. 2013;89(3):334-341.

7. Jimura K, Locke HS, Braver TS. Prefrontal cortex mediation of cognitive enhancement in rewarding motivational contexts. Proceedings of the National Academy of Sciences. 2010;107(19):8871-8876.

8. Krebs RM, Boehler CN, Woldorff MG. The influence of reward associations on conflict processing in the Stroop task. Cognition. 2010;117(3):341-347.

9. Christie ST, Schrater P. Cognitive cost as dynamic allocation of energetic resources. Frontiers in neuroscience. 2015;9:289.

10. Benoit CE, Solopchuk O, Borragán G, Carbonnelle A, Van Durme S, Zénon A. Cognitive task avoidance correlates with fatigue-induced performance decrement but not with subjective fatigue. Neuropsychologia. 2019;123:30-40.

11. Shenhav A, Musslick S, Lieder F, Kool W, Griffiths TL, Cohen JD, et al. Toward a rational and mechanistic account of mental effort. Annual review of neuroscience. 2017;40:99-124.

12. Yang F, Wu Q, Li S. Learning-induced uncertainty reduction in perceptual decisions is task-dependent. Frontiers in human neuroscience. 2014;8:282.

13. Tang YY, Posner MI. Attention training and attention state training. Trends in cognitive sciences. 2009;13(5):222-227.

14. Tang YY, Posner MI. Training brain networks and states. Trends in cognitive sciences. 2014;18(7):345-350. 
15. Petersen SE, Posner MI. The attention system of the human brain: 20 years after. Annual review of neuroscience. 2012;35:73-89.

16. Tang YY, Ma Y, Wang J, Fan Y, Feng S, Lu Q, et al. Short-term meditation training improves attention and self-regulation. Proceedings of the National Academy of Sciences. 2007;104(43):17152-17156.

17. Buschkuehl M, Jaeggi SM, Jonides J. Neuronal effects following working memory training. Developmental cognitive neuroscience. 2012;2:S167-S179.

18. Jensen CG, Vangkilde S, Frokjaer V, Hasselbalch SG. Mindfulness training affects attention - or is it attentional effort? Journal of Experimental Psychology: General. 2012;141(1):106.

19. Henson R, Rugg M. Neural response suppression, haemodynamic repetition effects, and behavioural priming. Neuropsychologia. 2003;41(3):263-270.

20. Kornmeier J, Pfaeffle M, Bach M. Necker cube: stimulus-related (low-level) and percept-related (high-level) EEG signatures early in occipital cortex. Journal of vision. 2011;11(9):12-12.

21. Hramov AE, Maksimenko VA, Pchelintseva SV, Runnova AE, Grubov VV, Musatov VY, et al. Classifying the perceptual interpretations of a bistable image using EEG and artificial neural networks. Frontiers in neuroscience. 2017;11:674.

22. Hramov AE, Frolov NS, Maksimenko VA, Makarov VV, Koronovskii AA, Garcia-Prieto J, et al. Artificial neural network detects human uncertainty. Chaos: An Interdisciplinary Journal of Nonlinear Science. 2018;28(3):033607.

23. Maksimenko VA, Runnova AE, Zhuravlev MO, Makarov VV, Nedayvozov V, Grubov VV, et al. Visual perception affected by motivation and alertness controlled by a noninvasive brain-computer interface. PloS one. 2017;12(12).

24. Leopold DA, Wilke M, Maier A, Logothetis NK. Stable perception of visually ambiguous patterns. Nature neuroscience. 2002;5(6):605-609.

25. Nuwer MR, Comi G, Emerson R, Fuglsang-Frederiksen A, Guérit JM, Hinrichs H, et al. IFCN standards for digital recording of clinical EEG.

Electroencephalography and clinical Neurophysiology. 1998;106(3):259-261.

26. Delorme A, Makeig S. EEGLAB: an open source toolbox for analysis of single-trial EEG dynamics including independent component analysis. Journal of neuroscience methods. 2004;134(1):9-21.

27. Maris E, Oostenveld R. Nonparametric statistical testing of EEG-and MEG-data. Journal of neuroscience methods. 2007;164(1):177-190.

28. Gramfort A, Luessi M, Larson E, Engemann DA, Strohmeier D, Brodbeck C, et al. MEG and EEG data analysis with MNE-Python. Frontiers in neuroscience. 2013;7:267.

29. Linden DE. The working memory networks of the human brain. The Neuroscientist. 2007;13(3):257-267.

30. Rahm B, Kaiser J, Unterrainer JM, Simon J, Bledowski C. fMRI characterization of visual working memory recognition. NeuroImage. 2014;90:413-422. 
31. Berger B, Griesmayr B, Minarik T, Biel A, Pinal D, Sterr A, et al. Dynamic regulation of interregional cortical communication by slow brain oscillations during working memory. Nature communications. 2019;10(1):1-11.

32. Zumer JM, Scheeringa R, Schoffelen JM, Norris DG, Jensen O. Occipital alpha activity during stimulus processing gates the information flow to object-selective cortex. PLoS biology. 2014;12(10).

33. Baumgarten TJ, Schnitzler A, Lange J. Prestimulus alpha power influences tactile temporal perceptual discrimination and confidence in decisions. Cerebral Cortex. 2014;26(3):891-903.

34. Anderson K, Ding M. Attentional modulation of the somatosensory mu rhythm. Neuroscience. 2011;180:165-180.

35. Bauer M, Kennett S, Driver J. Attentional selection of location and modality in vision and touch modulates low-frequency activity in associated sensory cortices. Journal of neurophysiology. 2012;107(9):2342-2351.

36. Linkenkaer-Hansen K, Nikulin VV, Palva S, Ilmoniemi RJ, Palva JM. Prestimulus oscillations enhance psychophysical performance in humans. Journal of Neuroscience. 2004;24(45):10186-10190.

37. Boksem MA, Kostermans E, Tops M, De Cremer D. Individual differences in asymmetric resting-state frontal cortical activity modulate ERPs and performance in a global-local attention task. Journal of Psychophysiology. 2012;.

38. Dale G, Arnell KM. Investigating the stability of and relationships among global/local processing measures. Attention, Perception, \& Psychophysics. 2013;75(3):394-406.

39. Gable PA, Poole BD, Cook MS. Asymmetrical hemisphere activation enhances global-local processing. Brain and Cognition. 2013;83(3):337-341.

40. Harmon-Jones E, Gable PA. Neural activity underlying the effect of approach-motivated positive affect on narrowed attention. Psychological Science. 2009;20(4):406-409.

41. Pitchford B, Arnell KM. Self-control and its influence on global/local processing: An investigation of the role of frontal alpha asymmetry and dispositional approach tendencies. Attention, Perception, \& Psychophysics. 2019;81(1):173-187.

42. Pitchford B, Arnell KM. Resting EEG in alpha and beta bands predicts individual differences in attentional breadth. Consciousness and cognition. 2019;75:102803.

43. Boksem MA, Meijman TF, Lorist MM. Effects of mental fatigue on attention: an ERP study. Cognitive brain research. 2005;25(1):107-116.

44. Barwick F, Arnett P, Slobounov S. EEG correlates of fatigue during administration of a neuropsychological test battery. Clinical Neurophysiology. 2012;123(2):278-284.

45. Vossel S, Geng JJ, Fink GR. Dorsal and ventral attention systems: distinct neural circuits but collaborative roles. The Neuroscientist. 2014;20(2):150-159. 
46. Spagna A, Martella D, Fuentes LJ, Marotta A, Casagrande M. Hemispheric modulations of the attentional networks. Brain and cognition. 2016;108:73-80.

47. Spagna A, Kim TH, Wu T, Fan J. Right hemisphere superiority for executive control of attention. Cortex. 2018;

48. Spagna A, Mackie MA, Fan J. Supramodal executive control of attention. Frontiers in psychology. 2015;6:65.

49. Myers NE, Rohenkohl G, Wyart V, Woolrich MW, Nobre AC, Stokes MG. Testing sensory evidence against mnemonic templates. Elife. 2015;4:e09000.

50. Rauss K, Pourtois G. What is bottom-up and what is top-down in predictive coding? Frontiers in psychology. 2013;4:276.

51. Wiggs CL, Martin A. Properties and mechanisms of perceptual priming. Current opinion in neurobiology. 1998;8(2):227-233.

52. Henson RN, Price CJ, Rugg MD, Turner R, Friston KJ. Detecting latency differences in event-related BOLD responses: application to words versus nonwords and initial versus repeated face presentations. Neuroimage. 2002;15(1):83-97.

53. Kourtzi Z, Kanwisher N. Cortical regions involved in perceiving object shape. Journal of Neuroscience. 2000;20(9):3310-3318.

54. Naccache L, Dehaene S. The priming method: imaging unconscious repetition priming reveals an abstract representation of number in the parietal lobes. Cerebral cortex. 2001;11(10):966-974.

55. Wagner AD, Koutstaal W, Maril A, Schacter DL, Buckner RL. Task-specific repetition priming in left inferior prefrontal cortex. Cerebral Cortex. 2000;10(12):1176-1184.

56. Theves S, Chan JS, Naumer MJ, Kaiser J. Improving audio-visual temporal perception through training enhances beta-band activity. NeuroImage. 2020;206:116312.

57. Arnal LH, Giraud AL. Cortical oscillations and sensory predictions. Trends in cognitive sciences. 2012;16(7):390-398.

58. Fujioka T, Trainor LJ, Large EW, Ross B. Internalized timing of isochronous sounds is represented in neuromagnetic beta oscillations. Journal of Neuroscience. 2012;32(5):1791-1802.

59. Bernasconi F, Manuel AL, Murray MM, Spierer L. Pre-stimulus beta oscillations within left posterior sylvian regions impact auditory temporal order judgment accuracy. International Journal of Psychophysiology. 2011;79(2):244-248. 